\title{
Integrated Optimization of Mechanisms with Genetic Algorithms
}

\author{
Jean-Luc Marcelin \\ Laboratoire G-SCOP, Université de Grenoble, Domaine universitaire, Grenoble, France \\ E-mail: Jean-Luc.Marcelin@uif-grenoble.fr \\ Received January 27, 2010; revised March 6, 2010; accepted March 9, 2010
}

\begin{abstract}
This paper offers an integrated optimization of mechanisms with genetic algorithm, the principle of which is to use a neural network as a global calculation program and to couple the network with stochastic methods of optimization. In other words, this paper deals with the integrated optimization of mechanisms with genetic algorithms, and, in conclusion, the possible use of neural networks for complex mechanisms or processes.
\end{abstract}

Keywords: Optimization, Mechanisms, Genetic Algorithm

\section{Introduction: The Need for an Integrated Optimal Design Process}

The search for the best compromise between economic, mechanical and technological imperatives has always been the primary aim of the mechanical engineer. The methods used to achieve these excellence objectives have considerably evolved over the last years. The author's experience in optimization began in 1983. At this time, the design stage was the main concern, and then came the calculation and afterwards the optimization. In practice, and during shape optimization of mechanical structures, occurred between 1985 and 1990, many extreme cases were encountered. In these cases, the optimization was not necessary until damage occurred during service; the author's industrial partners realized, often too late, that their designing left quite a bit to be desired. They would then call for the author's help in using optimization programs to supply them with an improved shape. These shapes were reached despite technological limitations being very severe at this stage; so severe, in fact, that engineers were powerless to resolve the problem. Innumerable problems such as this were dealt with.

Such an approach to designing has become unthinkable these days. The economic competition has increased, the design and manufacture delays have been reduced and therefore the numerous overlaps that this approach involves have become prohibitive. That is, optimization can no longer be distinct from designing. It is now admitted that, in an integrated design approach, optimization has to begin from the design stage, taking into account the constraints both in terms of specification and those induced by different materials. Optimization is therefore facilitated because constraints or limitations can be more easily varied, in agreement with all those involved with the project.

This paper aims at showing that the integration of optimization from the design phase is possible thanks to the new optimization techniques, according to the author. Some optimization methods are popular for now, they are known as probabilistic or stochastic. For example, the simulated annealing method or genetic algorithms, whose principle advantages are an assured convergence without the use of derivatives and eventual functions with discrete and non-derivable variables, even though determinist methods of optimization (called gradient methods) require a calculation resistant to these sensitivities. Genetic algorithms rely on the natural laws of selection which let a living organism adapt to a given environment. From these principles, it seems judicious to apply genetic algorithms to the optimization of mechanical structures. Precise examples will show that genetic algorithms will allow the adaption of the mechanical object to its environment and to the specifications, from the beginning of the design process.

\section{State of the art}

In [1], an integrated design optimization combining the mechanism method with genetic algorithms is presented. The former is currently one of the principal arch assessment tools; the latter are powerful numerical function optimization techniques. The method is proposed as a design aid for structural engineers involved in the assessment, maintenance and repair of existing bridges, or the design of new arches. In [2], fully integrated design optimization of plate structures is presented. First, to- 
pology optimization is introduced based on a hybrid algorithm, then fitting optimization is applied. Finally, the sizing optimization is described. In [3], the research tries to define the class of perfectly flexible mechanism. It proposes a generalized design methodology of perfectly flexible mechanism, and attempts to explore the technique of integrated mechanism for achieving the most optimal design alternative. In [4], a novel optimization approach to the design of mechanisms in morphing aircraft structures is presented. The layout of the mechanism and the location and number of actuators and pivots are determined by an extended formulation of a material-based topology optimization. The design problem is modeled within a coupled fluid-structure analysis framework to directly assess aerodynamic performance criteria while optimizing the overall mechanized system. In [5], it is said that the integrated design of missile weapon system is quite complicated, which challenges the sharing and optimization of models and design parameters. The paper first presents the conception of virtual collaborative design space on the basis of technology of virtual prototype and computer supported cooperative work, and then brings forward the architecture of virtual collaborative studio for integrated missile design and optimization.

On one hand, after introducing the methods and tools used (in part 2), this paper focuses on applications in the field of mechanical technology, and then on the analysis of mechanical systems (part 3). The examples of part 3 are to illustrate that the integrated optimization of mechanical structures has become a reality.

On the other hand, the conclusion explains why the difficulties are more important in the case of an integrated, optimal design process of mechanical systems, because of the complexity of the problems. Nevertheless, an integrated optimization can effectively be considered with the use of neural networks. Therefore, the conclusion provides a possible solution for the whole problem.

\section{The Methods Used: Adaptation of Optimization Tools to Mechanical Technology}

In addition to the above-mentioned elements, the author's experience began with the shape optimization of mechanical structures (2-D and symmetrical), although this was in the context of conventional design. See [6,7].

Mathematical optimization programs were quite difficult to use and not sufficiently versatile to adapt quickly to new cases. According to author, the optimal integrated design could not be achieved with normal mathematical programming techniques, which require a formulation heavily specific to each given problem. This paper aims at showing that stochastic techniques are ideally suited to integrated optimization and to mechanical technology problems.
Note that the problems' main features are the following:

- the design variables are often a mixture of discrete and continuous values;

- they are often highly constrained by strict technological constraints.

The problem consists in maximizing a function of $n$ variables. The principle of genetic algorithms is to make a population of individuals evolve according to a replica of Darwinian theories. The starting point is a population of individuals, chosen randomly and coded by binary numbers (as an example) called chromosomes. From this point, the algorithm generates, more or less randomly, new populations formed from individuals, increasingly more adapted to a given, well-defined environment. Selections and reproductions are made from the best performing parents of the population from which they come. They are stochastic or deterministic. These offsprings are generated applying genetic operators (mutation, crossing). It is always stochastic. The new replacement population is created by the selection of the best performing individuals, either among the offspring or its parents. The substitution is either stochastic or deterministic. In the books [8-11], additional information can be found, and the convergence of the method is shown.

The main advantage of these methods is that they operate simultaneously on a test space of the solutions. The genetic method differs from the simulated annealing method, because of the operators which are used to force the evolution of the test population. In all cases, the convergence is always assured towards an extreme. This extreme is not necessarily the absolute extreme, but has more chance of being so, than with the use of a traditional gradient method. This is shown in [8]. Actually, a stochastic method explores a larger solution space. In addition, another crucial advantage of these methods is the small number of required assumptions for the objective function. Genetic algorithms are now well-known.

\section{Neural networks}

We are to study the possible use of neural networks in the conclusion. The operation of artificial neural networks, as their name suggests, comes from that of biological neural networks, from which lots of terms have been borrowed. To the author, this is as far as the similarities go. More details on the theory, which will be summarized later, can be found in [12]. The use of neural networks for the simulation or modeling will be done in two stages: one phase called apprenticeship, using finite elements calculations-for example in mechanics of structures-and then a calculation or generalization phase. In the present case, neural networks should be able to estimate an objective function or a cost function of entry or design variables. It should be noted here that the entry variables will be the binary digits of the chromosomeswhen using a G.A.-or the real values of the design variables-when using the simulated annealing method. 
The apprenticeship phase consists in optimizing or adapting, through an apprenticeship rule and by modifying the weights at each link. To do this, an apprenticeship sample is used, that is solutions which will be previously determined by finite element analysis, for example in mechanics of structures. The principle criterion is to have a minimal error for the evaluation of the function. Local adaption rules (for which the weight optimization is based on the states of the neurons connected to corresponding links) are distinguished from other rules, which are much more difficult to put into use. The best known local rules, for which details can be found in [12], are the ones called supervised or non-supervised, and the iterative rules.

To summarize, a neural network works in two phases. In the first place, the apprenticeship phase, during which the adaption function is active. This allows the weight values to be optimized from a set of entry values (the design or conception variables) and from exit values (the objective function(s) or cost function) called the apprenticeship set. Then, the values of the weights are fixed during the second phase that is the calculation or generation mode. This allows the calculation by the neural network of the exit values, as a function of the entry values.

The results obtained bring a lot of hope in applying neural networks to modeling, especially for the simulation of the calculations for mechanical structures. See [13] and [14]. The continuation to modeling seems natural, as the action of modeling a process or a behavior requires knowing the principle characteristics of the process or behavior. The network knows how to extract these characteristics and can therefore be memorized easily.

\section{Integrated Optimal Design of Particular Mechanical Systems Optimization of Gears}

Gears are very complicated components. A large number of dominating factors vary in every case: radius of curvature, unitary loading, pressure, slip speeds, etc.... In some cases, the design variables are huge and take very discrete values (such as the module, the choice of materials). Often, several objectives work in competition: balancing the energy transmission in bending and under pressure, optimization of masses, balancing the slips, to mention only a few. The idea consists of automatically dimensioning a right-sided cylindrical gear or helical gear, so as to find a good compromise between a minimum weight, dynamic performance (energy transmission) and geometric criteria such as balancing the slips. This optimization problem is very difficult to solve by hand and often leads to compromised solutions that are not entirely satisfactory, therefore the idea of an automatic optimization technique is most desirable for this complex problem. The mathematical optimization methods depend on the understanding of objective function gradients and are difficult to adapt to gears for three main reasons:

1) first, some design variables are continuous while others are discrete,

2) then, derived programming is quite delicate because the optimizing functions often depend implicitly on the design variables,

3) finally, the major flaw is that these methods become blocked at a local extreme (often, the only way to pursue the program is to rerun the calculation from a new starting point). As all specialists must know, the optimization of gears has numerous solutions and often it is better to adapt them to given situations. Therefore, this leads to the use of a genetic algorithm to solve the problem. The problem of gear optimization is illustrated in Figure 1.

This problem presents two main difficulties. First of all, coding the solution in the form of a simple and efficient chromosome is complicated; so is finding a good compromise according to different objectives between the different criteria (weight, power differential, balancing slips).

The coded parameters are restrained so the field of study is not too large, and therefore the chromosome is not too long. In this way, we have not considered all of the design parameters in gearing, but only six main parameters: $\mathrm{k}, \mathrm{z} 1, \mathrm{x} 1, \mathrm{x} 2, \mathrm{~m}_{\mathrm{n} 0}$, and the material; other parameters such as the helix angle are fixed during the optimization. In such a case, this choice can be modified without any problems (Figure 1).

When selecting the objective function, we use a multi-objective technique where the objective function will actually be a balanced sum of the different functions that we want to obtain, such as the minimum weight and minimum difference between slips. In this case, we must compare some objectives with others; the effective choice can easily be reset, in the case of the same script for different objectives (the shape under which they appear is chosen by the researcher in the field of optimiza tion). Above all though, the difficulty consisted in choosing the weighting of the coefficients, in respect to

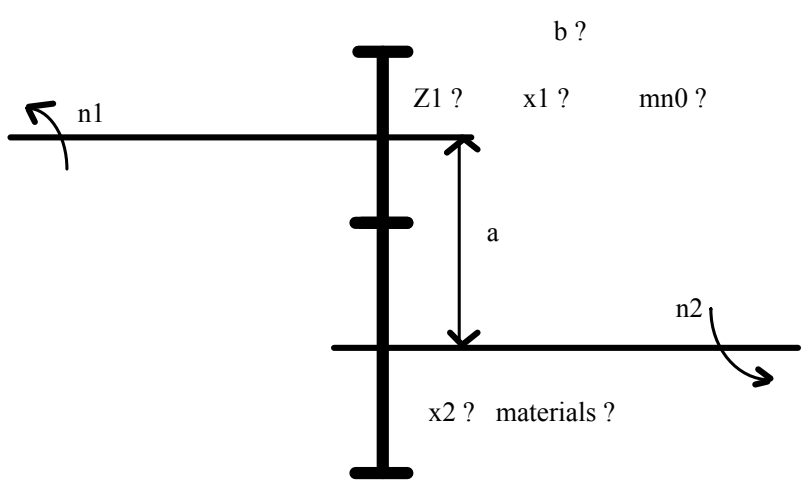

Figure 1. Definition of optimizing gearing. 
their influence (that are of different natures). This can only be done as a result of numeric experiments, where the goal was to find the best possible compromise between various objectives.

In the first place, the coding of the variables that we used in the genetic algorithm is the following: each of the values: $\mathrm{k}, \mathrm{z} 1, \mathrm{x} 1, \mathrm{x} 2, \mathrm{~m}_{\mathrm{n} 0}$ and materials are encoded in a binary numeration system. So, six strings are obtained C1, C2, C3, C4, C5, C6, lengthing 4, 6, 6, 7, 4, 3 respectively.

An example of a genetic identity card (chromosome) for a gearing system is given here.

Genetic identity coding (chromosome) for gearing:

100111010110110010101001001010

C1 $\quad$ C2 $\quad$ C3 $\quad$ C4 4 C5 $\quad$ C6

$\mathrm{C} 1$ : size coefficient of tooth ' $\mathrm{k}$ ',

$\mathrm{C} 2$ : number of teeth ' $\mathrm{Z} 1$ ',

$\mathrm{C} 3$ : coefficient ' $\mathrm{x} 1$ ',

C4: coefficient ' $\mathrm{x} 2$ ',

C5: real shape module ' $\mathrm{m}_{\mathrm{n} 0}$ ',

C6: material chosen from a library of 8 different types.

This coding is limited to a thirty-gene-long chromosome, and the genes are arranged end to end (the order is not important), so is the relative information of the gearing. This coding restrains the admissible field of design itself. There are only $2^{4}=16$ possible width ' $\mathrm{k}$ ' coefficients; only $2^{6}=64$ possibilities for the number of teeth ' $z 1$ ' (that can vary between 12 and 75 for example); $x 1$ and $\mathrm{x} 2$ only vary between -0.5 and 0.5 with two significant numbers; for $\mathrm{m}_{\mathrm{n} 0}$ there are 16 normalized numbers possible; finally, the material is the same for the pinion and for the gearwheel, and eight possible choices can be selected from the library of materials. For example, the code 001 corresponds to 30CND8, the code 110 to $16 \mathrm{NC6}$, and so on. It is thereafter possible to modify the structure or the length of the chromosome without too much difficulty.

In the second place, 'multi-objective' functions, in the case of gearing, are rather complicated. According to $\mathrm{u}$, the following allows us to modify at will, according to the results of diverse numerical experiments. The idea is to build the function as if it were the sum of the weighted representative terms, by coefficients that we can have varied when we wish, more or less according to the importance of such and such a criterion. The function that we used for the following tests is illustrated below:

$$
\begin{aligned}
& \mathrm{F}=10^{10}-\frac{\mathrm{I}_{1}}{\operatorname{Rap}}\left(\frac{\mathrm{b}}{\mathrm{b}_{\text {max }}}\right)\left(\frac{\mathrm{d}_{1}}{\mathrm{~d}_{1 \max }}\right)^{2}-\mathrm{I}_{2}\left|\mathrm{~g}_{\mathrm{s} 1}-\mathrm{g}_{\mathrm{s} 2}\right| \\
& -\mathrm{I}_{3} \frac{\mathrm{Rap}}{\mathrm{P}_{\text {trans }}}\left[\mid \mathrm{Pr}_{\mathrm{r} \text { up }}-\text { c.P } P_{\text {trans }}|+| \mathrm{P}_{\text {pres }}-\text { c.P } P_{\text {trans }} \mid\right]
\end{aligned}
$$

$\mathrm{I}_{1}, \mathrm{I}_{2}$ and $\mathrm{I}_{3}$ : weighting coefficients,

$\mathrm{g}_{\mathrm{s} 1}, \mathrm{~g}_{\mathrm{s} 2}$ : maximum absolute slips,

Rap: ratio of quality against price of material,

b: width of material, $\mathrm{d}_{1}$ : primitive diameter of pinion.

The presence of the term $10^{10}$ is due to the fact that the G.A. maximizes the functions. To calculate the functions at a minimum, it is possible to look for the maximum of the opposing function plus a very large term. The second term affected by coefficient $I_{1}$ is a term relating to the minimization of gear size, in relation to a given maximum size. This term is penalized when it comes to the quality/price ratio of a material. The third term, affected by coefficient $\mathrm{I}_{2}$, expresses the equalizing of the absolute slip (crucial in reducing wear). Finally, the fourth term, affected by coefficient $\mathrm{I}_{3}$, is a term expressing the search for balance between the transmissible powers under pressure and under flexion, and that must also respect the safety factor with relation to power transmitted. It is possible to add other criteria to this multi-objective function, e.g., a term expressing maximization of driving relation, or a term ensuring imposed distances between axes are respected. After several numeric tests on a basic example, the below values of weighting coefficients were chosen for the following test case:

$\mathrm{I}_{1}=0.2$
$\mathrm{I}_{2}=0.1$
$\mathrm{I}_{3}=10$

This test deals with a helical gear used in a fixed axis gearbox.

The parameters of the G.A. are:

population size $=200$

number of generations $=100$

The results are compared to a reference solution, optimized using other methods.

The given factors are:

$\mathrm{P}_{\text {trans }}=400 \mathrm{KW}$

Nmax(input) $=1,485 \mathrm{rpm}$

transmission relationship $\mathrm{u}=6$

developing circle of teeth $\mathrm{b}=8^{\circ} 33^{\prime}$

Quality factor $\mathrm{Q}=6$

Life $\mathrm{H}=200,000$ hours

operating with negligible shock.

The following is the best solution of the last generation: Geometrical analysis : mn0 Z1 $\quad \mathrm{x} 1 \quad \mathrm{x} 2 \quad \mathrm{k}$ Material $\begin{array}{lllllll}\text { reference solution } & 5 & 26 & 0.44 & -0.45 & 32 & 16 \mathrm{NC} 6\end{array}$ $\begin{array}{lllllll}\text { genetic algorithm } & 6 & 23 & 0.10 & 0.09 & 13 & 16 \mathrm{NC} 6\end{array}$ reference solution genetic algorithm

$\begin{array}{lcc}\text { width b }(\mathrm{mm}) & 160 & 78 \\ \mathrm{~d}_{1}(\mathrm{~mm}) & 131.4 & 139.9 \\ \text { volume bd }{ }_{1} 2 & 2.7 \mathrm{E} 6 & 1.5 \mathrm{E} 6 \\ \mathrm{~g}_{\mathrm{s} 1} & 0.24 & 0.48 \\ \mathrm{~g}_{\mathrm{s} 2} & 0.33 & 0.30 \\ \mathrm{e}_{\mathrm{e}} & 1.63 & 1.85 \\ \mathrm{P}_{\text {flex }}(\mathrm{kW}) & 1100 & 760 \\ \mathrm{P}_{\text {pres }}(\mathrm{kW}) & 1000 & 740\end{array}$

The objectives have been achieved: that is, we obtained a correct balance between absolute slips $\mathrm{g}_{\mathrm{s} 1}$ and 
$\mathrm{g}_{\mathrm{s} 2}$ and powers with a sufficient safety factor. It is also notable that the volume of the gear in the genetic solution is clearly inferior to that of the reference solution.

Lots of same other tests have been conducted, and the optimization objectives are always successfully met. For all these tests, the material systematically selected (from a list of available materials) is the highest performing, that is 16NC6. In reality, amongst the final solutions, other perfectly acceptable solutions use less high perfomance steels, but we have chosen the best one each time.

\section{Optimization of mechanisms}

We are going to show that the evolutionary methods can also be very efficient for issues linked with the optimization of mechanisms. The present section focusses on the problem of optimization of mechanisms under the following restricting hypothesis: we remain within the scope of fixed topologies and we consider isostatic or slightly hyperstatic mechanisms. The main goal is to minimize the force transmitted in each connection; the design variables are the relative positions of the different connections in respect to each other; furthermore some technological limitations on overall size, or the exclusion of precise areas of the layout or space for the connections, must be respected. Actually, we are dealing with a first approach, aimed at showing that it is possible to optimize mechanisms using completely random (trial and error) and automatic methods of optimization.

We are now to study a mechanism (representing a mixer) which creates a transformation of movement, represented in Figure 2. It is composed of 3 solids S1, $\mathrm{S} 2, \mathrm{~S} 3$, and a fixed housing S0. S1 is an entry shaft with uniform rotation. It is connected to the housing by a horizontal axis pivotal connection (fixed rolling element bearing). It is connected to S2 by a free floating rolling element bearing on a horizontal axis. $\mathrm{S} 2$ is connected to the exit shaft S3 by a ball and socket joint (which is actually a basic fixed rolling element bearing on a vertical axis); the fact that S2 and S3 are linked by a ball and socket joint make the system isostatic. S3, linked to the mixer blades, is connected to the housing with a free floating rolling element bearing around a vertical axis. It is assumed that only parts 1 and 3 have external forces applied to the mechanism. The objective is once again to minimize the unknowns inter efforts inside the system, aiming at finding the dimensions with the least cost. In order to be able to calculate the forces transmitted in the different connections, and for the purposes of this calculation only, it is assumed that all the components of the external forces applied to parts 1 and 3 are $10 \mathrm{kN}$ and all the components of moments applied to 1 and 3 are $1 \mathrm{kNm}$. This being, a standard program of static analysis of mechanisms allows us to calculate, for a given configu-

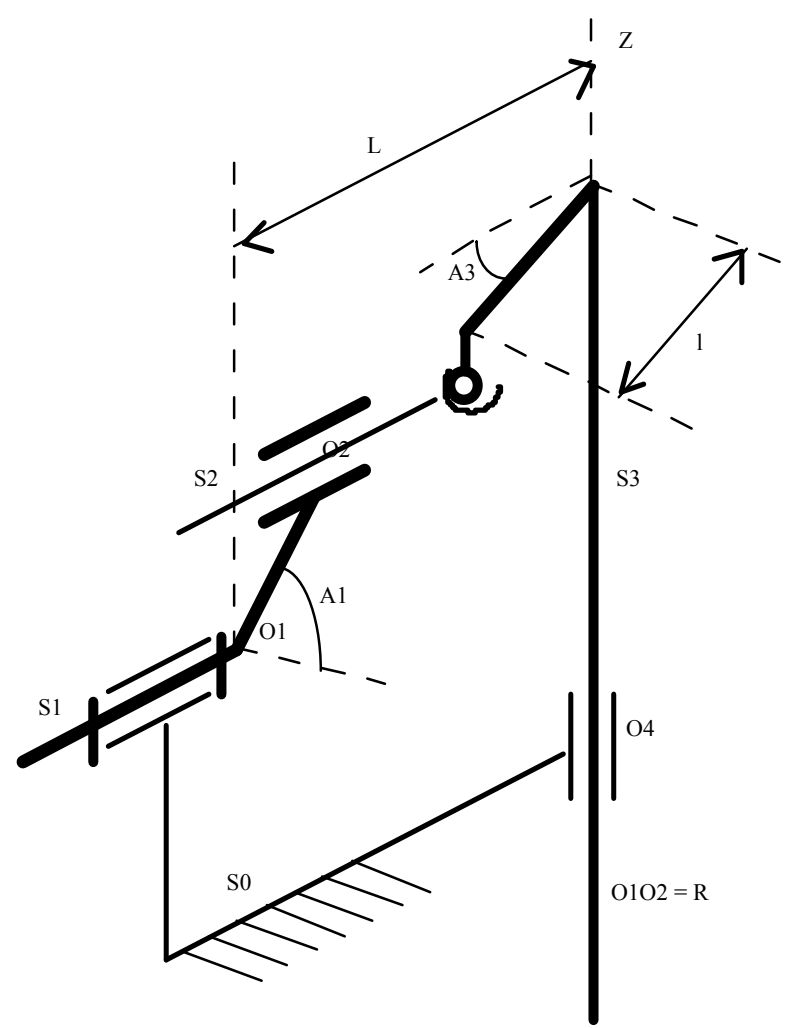

Figure 2. Optimization of mechanism.

ration of the mechanism, the torques transmitted in the different connections. For the purpose of this test, the objective function is taken to minimize the quadratic sum of all the components of forces and of moments of every connection (for this function to be homogenous, the moments are divided by an equal reference length, $100 \mathrm{~mm}$ ). When analyzing only 1 or 2 particular connections, the objective can be limited to only those components.

For the design variables that allow us to define the relative positions of connections with respect to one and other, 5, which are independent from each other, can be identified. These variables, marked X1 to X5 are the following:

$$
\begin{aligned}
& \mathrm{X} 1=1(\text { see Figure } 2) \\
& \mathrm{X} 2=\mathrm{R}=\mathrm{O} 1 \mathrm{O} 2 \\
& \mathrm{X} 3=\mathrm{z}(\mathrm{O} 4) \\
& \mathrm{X} 4=\text { angle } \mathrm{A} 1 \\
& \mathrm{X} 5=\text { angle } \mathrm{A} 2
\end{aligned}
$$

For the limitations on the design variables, the following factors are used:

- the horizontal dimension, $\mathrm{L}$, is fixed at a value of $\mathrm{L}$ $=200 \mathrm{~mm}$ (Figure 2); this limitation gives us a relationship that let us calculate the distance between $\mathrm{O} 2$ and the center of the ball and socket joint in terms of $\mathrm{L}, \mathrm{X} 1, \mathrm{X} 2$, $\mathrm{X} 4$, and $\mathrm{X} 5$.

Otherwise, the design variables are limited in the following manner: 


$$
\begin{gathered}
0<\mathrm{X} 1<100 \\
0<\mathrm{X} 2<50 \\
-100<\mathrm{X} 3<0 \\
0<\mathrm{X} 4<90^{\circ} \\
0<\mathrm{X} 5<45^{\circ}
\end{gathered}
$$

For this test, we must make an initial optimization using a G.A. to roughly work out the problem. The more precise optimization uses simulated annealing methods, starting from an initial solution given by the G.A.. Therefore, the optimization by the G.A. will be effective and, as it is only used for the first approximation, we limit the coding of the five design variables to a binary chromosome of 10 digits in total. The structure of this binary chromosome is the following:

- the first 2 digits allow the coding of variable $X 1$, the following 2 digits the coding of $\mathrm{X} 2$, and so on;

- the $2 \times 5$ digits are then put side by side to form a chromosome of 10 digits;

The coding is crude, but it is after an initial passage that can be improved using a more precise coding. In our case, the decoding will be done in the following manner:

variable X1: $\quad 00-->20$

$$
\begin{aligned}
& 01-->50 \\
& 10-->75 \\
& 11-->100
\end{aligned}
$$

variable X2: 00 --> 20

$$
\begin{aligned}
& 01-->30 \\
& 10-->40 \\
& 11-->50
\end{aligned}
$$

variable X3: $\quad 00-->-50$

$$
\begin{aligned}
& 01-->-60 \\
& 10-->-80 \\
& 11-->-100
\end{aligned}
$$

variable X4: $\quad 00-->0^{\circ}$

$$
\begin{aligned}
& 01-->20^{\circ} \\
& 10-->50^{\circ} \\
& 11-->90^{\circ}
\end{aligned}
$$

variable X5: $00-->0^{\circ}$

$$
\begin{aligned}
& 01-->10^{\circ} \\
& 10-->25^{\circ} \\
& 11-->40^{\circ}
\end{aligned}
$$

We can see that the limitations of the problem, in particular on the design variables are integrated in the coding. It is not necessary to penalize an objective function that will be of type a-F ("a" being a very large constant) because the G.A. maximizes the functions. For a population of 30 individuals and 50 generations, the G.A. quickly comes to the following solution:

$$
\begin{aligned}
& \mathrm{X} 1=100 ; \\
& \mathrm{X} 2=50 ; \\
& \mathrm{X} 3=-50 ; \\
& \mathrm{X} 4=20^{\circ} ; \\
& \mathrm{X} 5=0^{\circ} ;
\end{aligned}
$$

which corresponds to the chromosome 111000100 , and a value of $1.1013 \mathrm{E} 8$ for $\mathrm{F}$. This represents a gain of $30 \%$ in comparison to an average solution, for example:

$$
\begin{aligned}
& \mathrm{X} 1=20 ; \\
& \mathrm{X} 2=20 ; \\
& \mathrm{X} 3=-100 ; \\
& \mathrm{X} 4=90^{\circ} ; \\
& \mathrm{X} 5=40^{\circ} ; \\
& \text { Chromosome }=0000111111 ; \\
& \mathrm{F}=1.318 \mathrm{E} 8 ;
\end{aligned}
$$

We note that the solutions tend at taking $\mathrm{X} 1$ as large as possible and small angles A1 and A2, as far as the technology will allow. For the components of forces and moments, the results for the final solution and some force characteristics are given in the table below. Values for the average solution (chromosome 0000111111) are given in brackets.

for connection 01: Y01 = 200. DaN (753.), M01 = 4974. $\mathrm{mmDaN}$ (8467.)

for connection 12: Y12 = $1 . \mathrm{DaN}(6.5), \mathrm{M} 12=5025$. $\mathrm{mmDaN}$ (18467.), N12 = 5028. $\mathrm{mmDaN}$ (120522.)

for connection 23: $\mathrm{Y} 23=1 . \mathrm{DaN}(6.5)$

for connection 03: $\mathrm{Y} 03=0.1 \mathrm{DaN}$ (553.), $\mathrm{L} 03=$ 13386. $\mathrm{mmDaN}$ (89601.), $\mathrm{M} 03=6 . \mathrm{mmDaN}$ (8467.)

A very important reduction of the values of forces and moments can be noted.

\section{Conclusions: Towards an Optimal Integrated Design for Mechanical Systems}

As this paper showed, it is possible to aim at an optimal integrated design for mechanical structures. For now, the implementation of an optimal integrated design for mechanical systems, that is taking into account a maximum of information from the beginning (know-how, ability, optimization constraints), is difficult due to the fact that the necessary specialist software, in most cases, work independently from other programs. This can be illustrated by the example of a gear box. A program of mechanical analysis is used initially to ensure a sound structure from the start, afterwards, specialist software is used for calculations of gears, bearings, shafts,... The same applies for finite element calculations to control the shape and strength of certain components. Currently, even if each stage of the problem is presented in terms of optimization as part 3 showed (dealing with gears); the problems remain most of the time bound to a specific order. Research in integrated design is orientated towards the use of common databases at different stages of the design.

This work aims at offering a fundamentally different approach, allowing at once a both global and almost automatic optimization. It should be made clear that the point of view given here is that of a mathematician. The principle of the offered method is to use a neural network as a global calculation program, and to couple this network with stochastic methods of optimization. It is necessary to keep in mind that the new strategy proposed consists of three stages: first, defining the parameters of 
the mechanism taking stock of all design variables, as well as desired objectives and technological limitations; secondly, the "learning" of the neural network with the goal of having a "mega-program" of analysis and calculation (perfectly adapted to the task in hand), including knowledge of all the programs which will be used in the design process; finally, use of this "mega-program" for totally automatic optimization, without the need for human intervention, thanks to stochastic methods; the method used here is that of G.A.. The expected result is a play of optimal design variables. This strategy has been developed in [15] for gear boxes, and in [16] for hotrolled complex beams.

\section{References}

[1] D. M. Peng and C. A. Fairfield, "Optimal Design of Arch Bridges by Integrating Genetic Algorithms and the Mechanism Method," Engineering Structures, Vol. 21, No. 1, 1999, pp. 75-82.

[2] F. Belblidia and E. Hinton, "Fully Integrated Design Optimization of Plate Structures," Finite Elements in Analysis and Design, Vol. 38, No. 3, 2002, pp. 227-244.

[3] W. G. Shao, "Perfectly Flexible Mechanism and Integrated Mechanism System Design," Mechanism and Machine Theory, Vol. 39, No. 11, 2004, pp. 1155-1174.

[4] K. Maute and G. W. Reich, "Integrated Multidisciplinary Topology Optimization Approach to Adaptive Wing Design," Journal of Aircraft, Vol. 43, No. 1, 2006, pp. 253-263.

[5] S. Mei, W. Zhao and W. Wang, "A Virtual Collaborative Design Space for Integrated Missile Design," Journal of System Simulation, Vol. 16, No. 7, 2004, pp. 1509-1529.

[6] J. L. Marcelin and Ph. Trompette, "Optimal Shape Design of Thin Axisymmetric Shells," Engineering Optimization, Vol. 13, No. 2, 1988, pp. 109-117.

[7] V. Steffen and J. L. Marcelin, "On the Optimization of
Vibration Frequencies of Rotors," The International Journal of Modal Analysis, Vol. 3, No. 3, 1988, pp. 77-80.

[8] D. E. Goldberg, "Genetic Algorithm in Search, Optimization and Machine Learning," Addison-Wesley, 1989.

[9] J. R. Koza, "Genetic Programming: On the Programming of Computers by Means of Natural Evolution," MIT Press, Massachusetts, 1992.

[10] Z. Michalewicz, "Genetic Algorithms + Data Stuctures = Evolution Programs," 3rd Edition, Springer Verlag, New York, 1996.

[11] D. E. Rumelhart and J. L. McClelland, "Parallel Distributed Processing," MIT Press, Cambridge, Massachusetts, 1986.

[12] J. F. Joduin, “Les Réseaux Neuromimétiques," Hermès, 1994.

[13] Z. Szewczyk and P. Hajela, "Neurocomputing Strategies in Structural Design-Decomposition Based Optimization," Structural and Multidisciplinary Optimization, Vol. 8, No. 4, 1998, pp. 242-250.

[14] P. Hajela and Z. Szewczyk, "Neurocomputing Strategies in Structural Design-On Analysing Weigths of Feedforward Neural Networks," Structural and Multidisciplinary Optimization, Vol. 8, No. 4, 1994, pp. 236-241.

[15] J. L. Marcelin, “A Metamodel Using Neural Networks and Genetic Algorithms for Integrated Optimal Design of Mechanisms," International Journal of Advanced Manufacturing Technology, Vol. 24, No. 9-10, 2004, pp. 708-714.

[16] J. L. Marcelin, "Optimization of the Cooling Regime of Hot-Rolled Complex Beams," International Journal of Advanced Manufacturing Technology, Vol. 32, No. 7-8, 2007, pp. 711-718. 